\title{
A Novel Ion Trap That Enables High Duty Cycle and Wide $m / z$ Range on an Orthogonal Injection TOF Mass Spectrometer
}

\author{
Alexander V. Loboda and Igor V. Chernushevich \\ MDS Analytical Technologies, Concord, Ontario, Canada
}

\begin{abstract}
Although TOF analyzers with orthogonal ion injection provide the whole spectrum without scanning, their duty cycle is low compared with scanning analyzers in single ion monitoring mode. Typical duty cycle is in the range of $5 \%$ to $30 \%$ depending on the instrument geometry and ion $\mathrm{m} / \mathrm{z}$ value. We present here a novel trapping/releasing setup, which offers the duty cycle near $100 \%$ over a wide range. Operation in the mass range from $\mathrm{m} / \mathrm{z} 120$ to almost 2000 is demonstrated. Ions are trapped in a short linear ion trap at the end of the collision cell in an axial pseudopotential well created by additional rf ("AC") voltage applied to all four rods of the trap with the same amplitude and phase. The pseudopotential created by AC field is mass dependent, and by ramping down the AC voltage, ions can be released from the trap sequentially from high $\mathrm{m} / \mathrm{z}$ to low, while all ions are gaining the same kinetic energy. Upon entering the TOF accelerator, ions with lower $\mathrm{m} / \mathrm{z}$ catch up with heavier ions, and the AC ramp parameters can be selected to make all ions meet in the center of the TOF extraction region, resulting in sensitivity gains from 3 to 14 without loss of mass accuracy or resolution. (J Am Soc Mass Spectrom 2009, 20, 1342-1348) (c) 2009 American Society for Mass Spectrometry
\end{abstract}

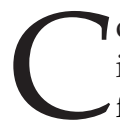
ontemporary TOF instruments with orthogonal injection (orthoTOF) [1-3] are widely recognized for their high-resolution, mass accuracy, sensitivity, and convenience of coupling to continuous ion sources. They are utilized in a range of fields today, either in the form of stand-alone TOF analyzers such as in ESI-TOF instruments [4], or as a final stage of mass analysis in hybrid quadrupole-TOF mass spectrometers $[5,6]$. Although orthoTOF provides the whole spectrum without scanning, it suffers from a relatively low sensitivity compared with scanning analyzers in single ion monitoring mode. A significant part of these losses is due to the low duty cycle of orthoTOF, typically $5 \%$ to $30 \%$, depending on the instrument geometry and ion $\mathrm{m} / \mathrm{z}$-value. Whitehouse et al. [7], and later Chernushevich [8] developed a method of trapping and subsequent timed release of ions into orthoTOF analyzer, which provides nearly $100 \%$ duty cycle, but only for a narrow $\mathrm{m} / \mathrm{z}$ range. Brenton et al. [9] demonstrated a significant improvement of the duty cycle using over-pulsing and ion gates inside the TOF drift region, but again for a small $\mathrm{m} / \mathrm{z}$ range. To increase $\mathrm{m} / \mathrm{z}$ range, Okumura et al. [10] proposed a "synchronous coupling of [a 3D-] trap and TOF," where, in the first step ions are geometrically prearranged according to their $\mathrm{m} / \mathrm{z}$ value in a combination of attractive DC field and repelling rf pseudopo-

Address reprint requests to Dr. I. V. Chernushevich, MDS Analytical Technologies, 71 Four Valley Dr., Concord, Ontario L4K4V8, Canada. E-mail: chernuiv@sciex.com tential, and in the second step ions are ejected orthogonally into TOF, and ions with lower $m / z$ catch up with heavier ions in the focal point ideally located in TOF accelerator. However, the spatial prearrangement is limited by the size of the ion trap, while the spatial spread in the presence of DC field makes the energy of ions leaving the trap $\mathrm{m} / \mathrm{z}$-dependent. In a different approach, Hashimoto et al. [11] achieved duty cycle larger than $60 \%$ over a mass range from $\mathrm{m} / \mathrm{z} 174$ to 1922 by using axially-resonant-excitation linear ion trap (LIT), which sequentially ejects ions according to their $\mathrm{m} / \mathrm{z}$ values into an adjacent non-mass-selective LIT in front of the TOF pusher. A delay between the ejection timing of the non-mass-selective LIT and the push timing of the orthoTOF was swept mass-synchronously with the axially-resonant-excitation LIT, thus extending the $\mathrm{m} / \mathrm{z}$ range of the method described earlier [7, 8]. Although this rather complicated set-up (essentially comprising two mass analyzers working as one) achieves high duty cycle for a wide mass range, its operation is rather limited by the $20 \mathrm{~Hz}$ repetition rate of the axially-resonant-excitation LIT.

We present here a novel trapping/releasing setup, which is free of most limitations mentioned above and offers the duty cycle near $100 \%$ over a wide $\mathrm{m} / \mathrm{z}$ range.

\section{Method}

All experiments were performed on a prototype quadrupole-TOF instrument shown schematically in Fig- 


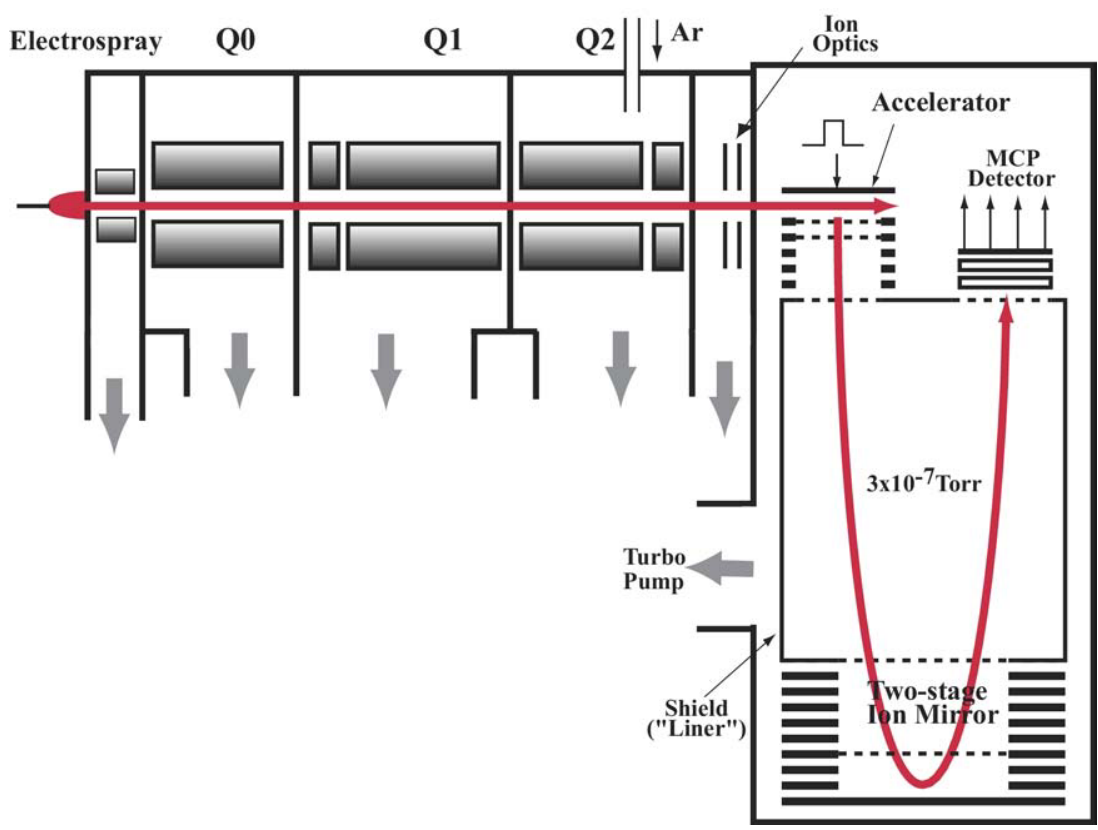

Figure 1. Schematic of the QqTOF mass spectrometer used in the present study.

ure 1. In brief, the QqTOF mass spectrometer consists of four quadrupoles: low vacuum quadrupole ion guide (QJet, MDS Analytical Technologies-Applied Biosystems), ion guide Q0, analyzing Q1 and collision cell Q2, and reflecting TOF with orthogonal injection. Combination of improved ion optics, $15 \mathrm{keV}$ acceleration voltage and a two-stage ion mirror provides mass resolution exceeding 20,000 (FWHM). Spectra were recorded with the 6-channel 50 ps resolution TDC (TC 890; Acqiris, Geneva, Switzerland), although only 4 TDC channels were used with a 4-anode detector.

Three versions of collision cells have been used in experiments. In all three cases, the quadrupole was built of rods $4.7 \mathrm{~mm}$ in diameter $(1 / 2$ of that of the Q0/Q1 rods), field radius $2.1 \mathrm{~mm}$, and $\sim 20 \mathrm{~cm}$ long; 3.5 $\mathrm{MHz}$ rf voltage applied in a quadrupolar mode provides radial confinement of ions in all Q2 including the trap. Four Linac II electrodes [12] create a weak axial field to accelerate ions through Q2. In the first version, a small linear ion trap (TR) was added at the exit end of collision cell Q2, as shown in Figure 2. It has the same rod diameter and field radius as the main Q2 rod set and is located in the same vacuum compartment, where the nitrogen pressure is maintained between 3 and 10 mTorr. Electrically, the $5 \mathrm{~mm}$ long segments of the trap are separated from the main rod set by a gas-transparent gating electrode designated historically as IQ3. The IQ4 electrode with a 2.4-mm aperture serves as an exit gate of the trap. In addition to the main rf and DC voltages, another rf (later called AC) voltage can be applied to all four trap electrodes in the same phase to provide axial trapping of ions. The AC frequency is selected to be half of the main $\mathrm{rf}$ frequency $(1.75 \mathrm{MHz})$ and is initiated by Agilent (Santa Clara, CA) 33220A function/arbitrary waveform generator. All pulsed and DC voltages related to the described method are supplied by home-made

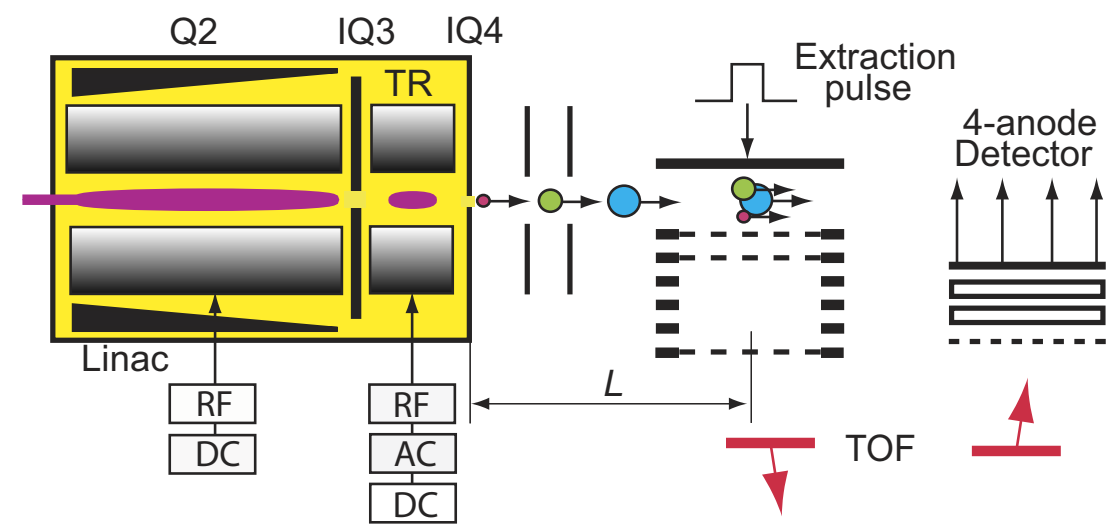

Figure 2. Schematic of the collision cell Q2 with a small linear ion trap at the end (version 1). 
electronics connected to 8-channel analog output board (NI-6733; National Instruments, Austin, TX) and controlled by sequencing software developed in-house.

The main difference of the second version of the trapping set-up is that the main rod set is extended to full length of the collision cell, penetrating through the first gating electrode IQ3. The trap is thus formed by the same gates IQ3 and IQ4, and by the last $5 \mathrm{~mm}$ of the main quadrupole, to which rf, AC, and DC voltages are supplied. The cross-section (Figure 3) made at the end of Linac shows the quadrupole rods, four Linac electrodes, IQ3 gating electrode behind the Linac, and the IQ4 exit aperture at the back. The rf and $\mathrm{AC}$ frequencies were increased in this version to $4.5 \mathrm{MHz}$ and $2.25 \mathrm{MHz}$ respectively. The main advantage of version 2 is that both mechanical assembly and electronics are simplified for this setup.

Finally, version 3 is mechanically identical to version 2 , but AC voltage is applied to the exit gate IQ4 rather than to the quadrupole rods. This set-up is the simplest of all three since there is no need to apply all of rf, AC, and DC voltages superimposed to the same electrodes. From a performance point of view, it is noteworthy that ions are affected by $\mathrm{AC}$ voltage even after they leave the collision cell.

Under normal continuous operation conditions, the ion beam leaves the collision cell Q2 through lenses IQ3 and IQ4 and enters TOF accelerator after being focused by ion optics. Ions move slowly (accelerated by $30 \mathrm{~V}$ ) through a slit into the accelerator. Short ion packets are formed in the extraction region of the accelerator, where "push" and "pull" pulses are applied to the plate and grid correspondingly. Finally, ion packets are accelerated by $-15 \mathrm{kV}$ applied to the last grid of accelerator

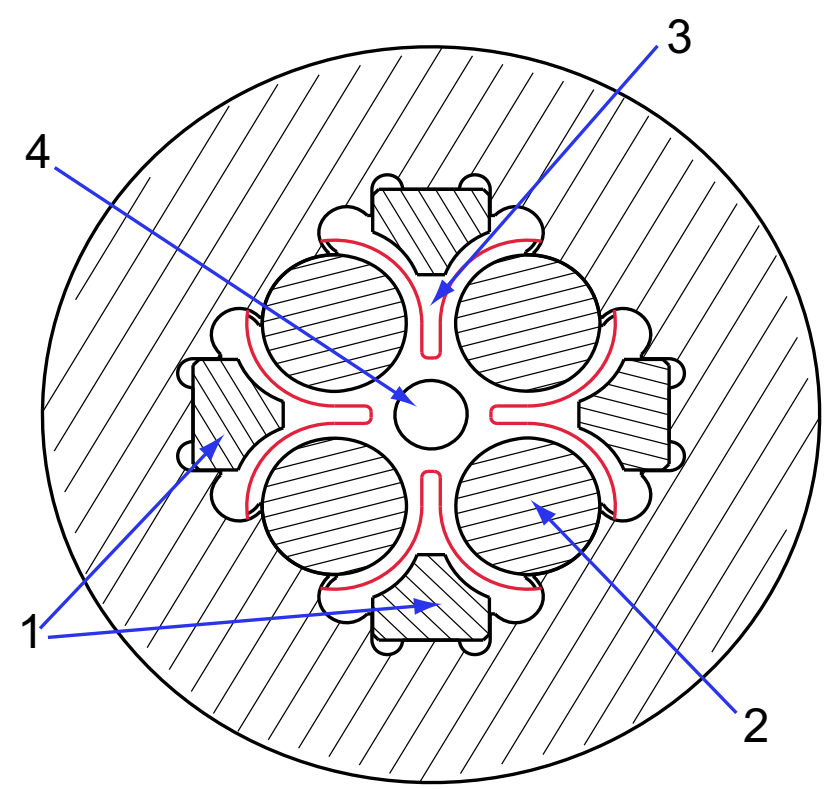

Figure 3. Cross-section of the collision cell at the end of Linac electrodes $3 \mathrm{~mm}$ before IQ3 (versions 2, 3). 1, Linac II electrodes; 2, quadrupole rods; 3, IQ3 gating electrode; 4, aperture in IQ4 exit electrode. into the drift region of the TOF mass analyzer. As discussed previously [8], in this mode of operation a continuous ion beam overfills the extraction region of the accelerator, and a large portion of ions is lost on the walls. Since the ion velocity component in the orthogonal direction is constant throughout the TOF analyzer, the largest duty cycle is equal to the ratio of the length of TOF entrance window $l=32 \mathrm{~mm}$ to the distance between mid-points of the accelerator and detector $b=$ $110 \mathrm{~mm}$. Therefore, for the heaviest ion the duty cycle is $29 \%$. Light ions move faster, and overfill the extraction region even more:

$$
\text { DutyCycle }=\frac{l}{b} \sqrt{\frac{(m / z)}{(m / z)_{\max }}}=29 \% \sqrt{\frac{(m / z)}{(m / z)_{\max }}}
$$

where $(m / z)_{\max }$ corresponds to the upper $m / z$ limit for a given spectrum. Formula 1 will be used later to backcalculate the largest possible gain from recovering the TOF duty cycle.

To eliminate the losses associated with continuous ion introduction, the new trapping/releasing method is utilized, which is illustrated by Figures 2 and 4 and involves the following steps:

1. A portion of ions is accumulated in the TR trap (IQ3 gate open, IQ4 gate closed).

2. A DC potential barrier is created at IQ3 gate, and ions accumulated in the trap are allowed to cool down to near thermal temperature, while the newly coming ions are preserved in the potential well formed by the Linac and IQ3 to be used in the next cycle.

3. An additional $\mathrm{rf}($ " $\mathrm{AC}$ ") voltage is applied to all four rods of the trap (or to IQ4 in version 3) with the same amplitude and phase to create on-axis pseudopotential well; the IQ4 voltage is switched from repelling to attractive, while ions still remain trapped due to the pseudopotential.

4. AC voltage is ramped down over a period of time $\tau$. Since the effective potential is $m / z$-dependent, ions are released in the order of decreasing $m / z$. The kinetic energy of the released ions is constant. Thus, the ion velocity is $m / z$-dependent, lighter ions moving faster than heavier ones. Selecting the correct ramp of the AC amplitude creates the condition where all ions arrive at the center of the TOF accelerator at the same time, independent of $\mathrm{m} / \mathrm{z}$.

The method is thus based on the fact that the pseudopotential created by AC (or rf) field is mass dependent, and that by ramping down the $\mathrm{AC}$ voltages ions can be released from the trap sequentially from high $\mathrm{m} / \mathrm{z}$ to low, while all ions are gaining essentially the same amount of kinetic energy defined by their acceleration in DC field after exiting the trap. The latter condition is very important since it enables ions across the whole $m / z$ range to follow the same trajectory inside the orthoTOF. 


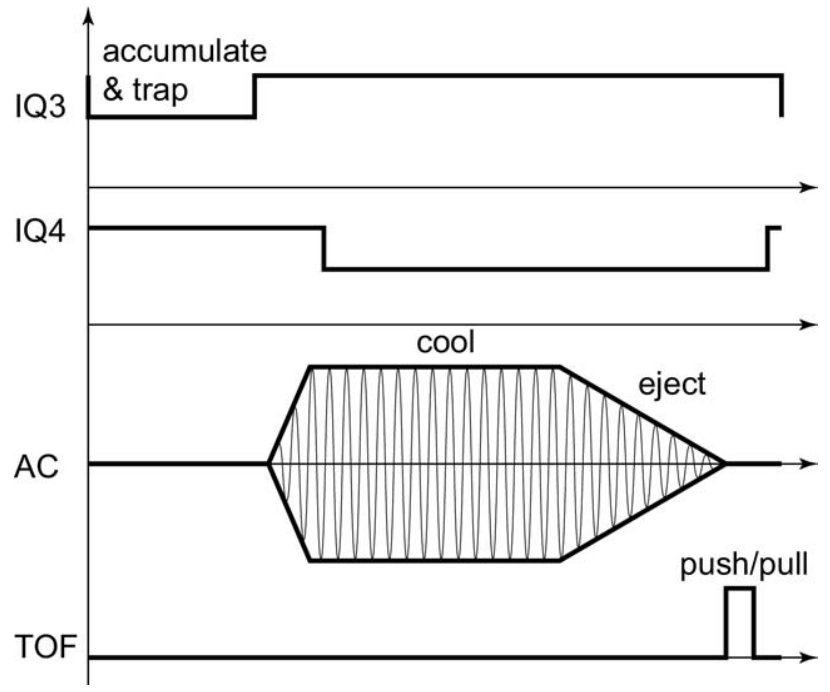

Figure 4. Timing diagram of gating voltages, AC ramp, and TOF extraction pulses.

\section{Brief Theory}

A simplified analytical treatment demonstrates that by choosing an appropriate slope of the AC ramp ions with different $\mathrm{m} / \mathrm{z}$ values can be brought together at a focal point in the desired location i.e., in the center of the extraction region of the accelerator.

The depth of the mechanical pseudopotential well created by inhomogeneous radio-frequency AC field in this configuration can be expressed as [13]:

$$
U_{p s e u d o}=z e \cdot C \frac{U_{A C}^{2}}{\omega^{2}(m / z)}
$$

where $e$ is the charge of electron, $U_{A C}$ is the AC voltage amplitude, $m$ and $z$ are ion mass and charge respectively, $\omega$ is the AC angular frequency, and $C$ is the geometry-dependent parameter. When an ion with a particular $m / z$ is about to leave the trap, the depth of the pseudopotential (2) becomes equal to the energy gain due to the DC voltage difference between TR and IQ4 electrodes; in other words the pseudopotential well is no longer deep enough to contain this ion:

$$
z e \cdot C \frac{U_{A C}^{2}}{\omega^{2}(m / z)} \approx z e \cdot(T R-I Q 4)
$$

where $T R$ and IQ4 are potentials of respective electrodes. Let us assume now that a linear AC ramp can satisfy the condition of bringing all ions into a focal point:

$$
U_{A C}=U_{0}(1-t / \tau)
$$

where $U_{0}$ is the largest AC amplitude and $\tau$ is the total ramp down time. The time $t_{1}$ an ion would spend in the trap since the beginning of the ramp at $t=0$ can be calculated as:

$$
t_{1}=\tau \cdot\left(1-\sqrt{\frac{(T R-I Q 4) \cdot \omega^{2} \cdot m / z}{C U_{0}^{2}}}\right)
$$

On the other hand, the time $t_{2}$ the same ion would spend in a subsequent flight over a distance $L$ (Figure 2) is equal to:

$$
t_{2}=L \sqrt{\frac{m / z}{2 e U_{\perp}}}
$$

where $U_{\perp}$ is the ion energy before TOF extraction. Equation 6 involves a significant simplification by assuming that the ion is immediately accelerated to the $U_{\perp}$ energy after exiting the TR region and that there are no fields on the way to the accelerator which is never true in the presence of ion optical electrodes. However, formula 6 gives a good approximation if we assign $L$ to be an "effective" flight length instead of the actual mechanical distance. To make light ions catch up with the heavy ones, the total time $t_{\Sigma}$ has to be independent of $m / z$ and equal to $t_{2}$ for ions with the largest $(m / z)_{\max }$ which are ejected at $t=0$ :

$$
\begin{aligned}
t_{\Sigma}= & t_{1}+t_{2}=\tau-\tau \sqrt{\frac{(T R-I Q 4) \cdot \omega^{2} \cdot m / z}{C U_{0}^{2}}} \\
& +L \sqrt{\frac{m / z}{2 e U_{\perp}}}=L \sqrt{\frac{(m / z)_{\max }}{2 e U_{\perp}}}
\end{aligned}
$$

To satisfy these conditions, the two terms containing $(\mathrm{m} / \mathrm{z})^{1 / 2}$ have to cancel each other, thus bringing us to the following solution:

$$
\begin{aligned}
\tau & =L \sqrt{\frac{(m / z)_{\max }}{2 e U_{\perp}}} \text { and } \\
U_{0} & =\sqrt{\frac{(T R-I Q 4) \cdot \omega^{2} \cdot(m / z)_{\max }}{C}}
\end{aligned}
$$

which defines both parameters of the AC ramp. The above formulae demonstrate that a linear $\mathrm{AC}$ ramp will result in gathering of all ions in a required focal point at the same time. In practice, however, accurate calculations of $\tau$ and $U_{0}$ are not always necessary because all that is needed to make ions arrive simultaneously in the desired location is the right slope of the ramp $\left(U_{0} / \tau\right)$, while $\tau$ and $U_{0}$ values just have to be large enough to cover the $\mathrm{m} / \mathrm{z}$ range of ions to be recorded.

This situation that causes light ions to chase the heavy ions reminds one of the ancient paradox of Achilles and the Tortoise described by Zeno of Elea [14] in the 400s BC. According to Zeno, Achilles, in a footrace with the tortoise, will never catch up with her if the tortoise has been given a head start. Although this 
study proves the opposite for ions, we now call the new method "Zeno pulsing" of ions.

\section{Results and Discussion}

Our first experiments were aimed at exploring the best operational conditions, as well as finding limitations of the new method. Trapping and releasing voltages had to be tuned interactively together with the $\mathrm{AC}$ ramp parameters. The maximum Zeno pulsing repetition rate was determined to be about $1.25 \mathrm{kHz}$, limited primarily by minimal required cooling time $(\sim 400 \mu \mathrm{s})$ and the length of the AC ramp ( $200 \mu \mathrm{s})$. It is worth mentioning here that the four-anode detector turned out to be very helpful during debugging and tuning of the setup. Since the four anodes (ca. $9 \times 30 \mathrm{~mm}$ each) are arranged consecutively along the axis of the original ion beam before it enters the TOF section, the ion intensity distribution over these anodes provides information on the spatial spread of the ion beam along that axis at the detector location. Under optimal tuning conditions, the intensity distribution is centered in the middle of the detector. Any shift of the ion intensity distribution points instantly to the need for adjusting the slope of the AC ramp and/or the delay. On the other hand, the width of this distribution gives an estimate of the length of ion packets after the TOF analysis. In continuous injection mode, ion distribution over anodes is typically 20\%-30\%-30\%-20\%, while in Zeno pulsing mode it usually shrinks to $10 \%-40 \%-40 \%-10 \%$, thus indicating that the ion beam is narrower in this mode. The narrow distribution of the ion beam in Zeno pulsing mode may explain why the sensitivity gains observed in this mode are sometimes slightly higher than those predicted by the formula 1 .

To evaluate the efficiency of the new method, two spectra were recorded for each compound tested: one with continuous ion introduction into extraction region, and another with Zeno pulsing. Switching between the two modes is done by selecting a state file in software, which automatically changes all involved voltages, triggers AC ramp and gating pulses, as well as changes TOF repetition rate, which is between 13 and $18 \mathrm{kHz}$ in normal mode and 1 and $1.25 \mathrm{kHz}$ in Zeno pulsing mode. Figure 5 shows two electrospray MS/MS spectra of a doubly charged ion of $\left[\mathrm{Glu}^{1}\right]$-fibrinopeptide $\mathrm{B}$ (Sigma-Aldrich, Oakville, Ontario, Canada) with $\mathrm{m} / \mathrm{z}=$ 785.8 recorded in two operational modes with collision energy of $54 \mathrm{eV}$ per charge. All other conditions were the same including the acquisition time. As evident from Figure 5, the new method leads to a significant gain in sensitivity, which is $m / z$-dependent: a factor of 14.5 at $m / z=120$ down to a factor of 4.2 at $m / z=1285$. The gains predicted by formula 1 are 15.5 and 4.8 , respectively. Experimental gain averaged over all fragment ions is about 7.

+TOF Product (785.8): Sample 3 (through, 1:100, lowR, RF100, CE=54eV) of GlufibB_ZenoTest_Dec11_06.wiff

Max. 414.0 counts.

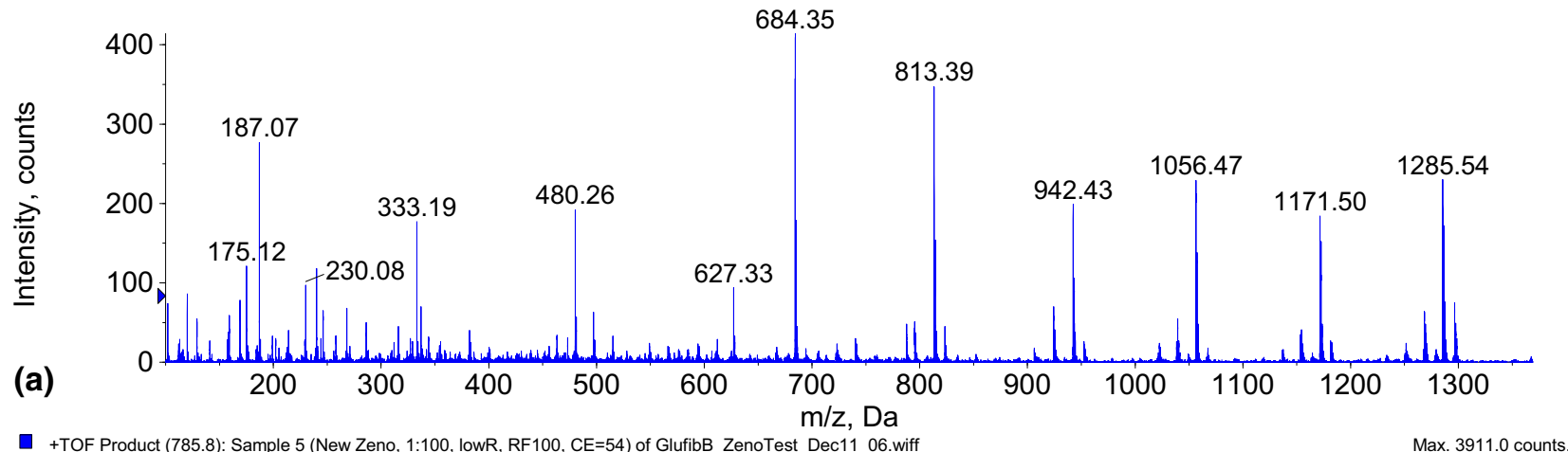

-TOF Product (785.8): Sample 5 (New Zeno, 1:100, lowR, RF100, CE=54) of GlufibB_ZenoTest_Dec11_06.wiff

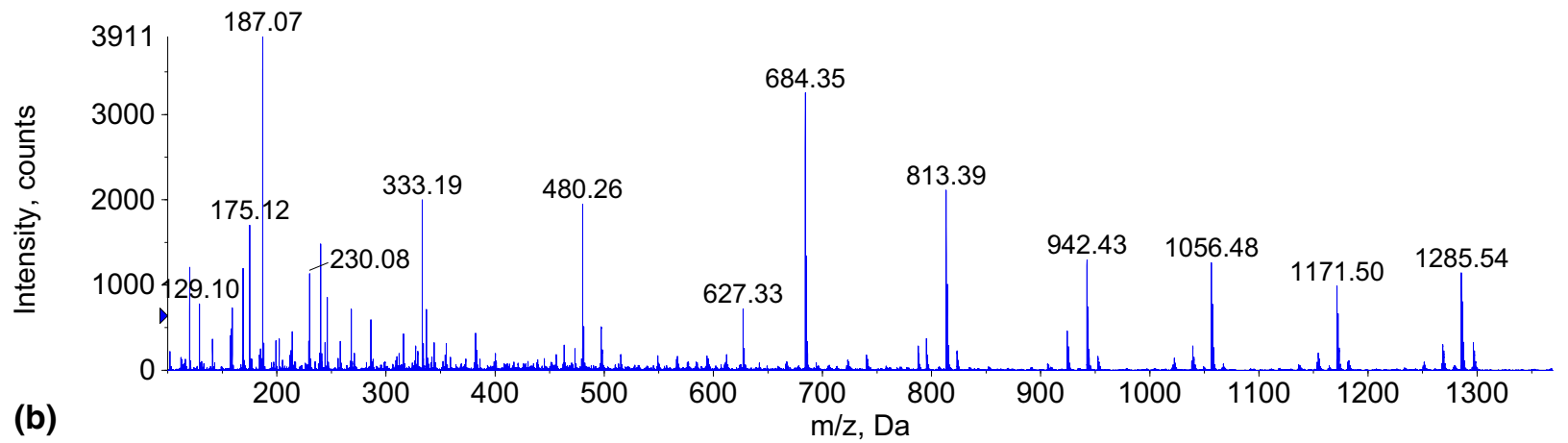

Figure 5. Electrospray fragment ion spectra of $2+$ ion of $\left[\mathrm{Glu}^{1}\right]$-fibrinopeptide $\mathrm{B}, \mathrm{m} / \mathrm{z}=785.8$ recorded in two operational modes: (a) normal continuous introduction mode, (b) Zeno pulsing mode (version 1). All other conditions were the same. 
Another test compound that was used for evaluation of all three versions of the method was peptide ACTH, fragment 18-39 (Sigma-Aldrich, Canada) with MW = 2464.2 Da. Upon fragmentation at $72 \mathrm{eV}$ collision energy (per charge), its doubly charged ion at $m / z=1233.1$ produces a rich spectrum with fragments in the range from $m / z 120$ to almost 2000. The plot of sensitivity gain (averaged over three experiments) versus $m / z$ is shown in Figure 6 for all three versions of collision cell, as well as the theoretical gain predicted by formula 1 . The plot shows that version 1 (with segmented trap, Figure 3) allows almost full restoration of duty cycle, except for light ions with $m / z<200$. Version 2 with AC applied to the main rod set brings average duty cycle up to about $88 \%$, presumably losing some ions during the trapping step. The smallest average gain $(\sim 76 \%$ of theoretical) was obtained with version 3 where $A C$ is applied to the exit gate IQ4. These efficiency measurements are in good agreement with the observed detector anode distributions, from which a rough estimate of the width of spatial distributions of ions at the detector plane can be obtained. The estimated width of this distribution increases from version 1 (ca. $20 \mathrm{~mm}$ ) to version 2 (25 to $35 \mathrm{~mm}$ ), and finally to version 3 (35 to $40 \mathrm{~mm}$ ). For comparison, the diameter of the extraction window (top grid in Figure 2) is equal to $32 \mathrm{~mm}$.

When modifying a TOF instrument with relatively high mass resolution and accuracy, it is important to study what effect the concomitant ion beam manipulations have on these critical parameters, or whether any TOF voltages require re-tuning. It was a pleasant surprise that no re-tuning was required when switching to trap/ramp mode, and resolution typically improved by $\sim 10 \%$ in versions 1 and 2, for example from average 20,000 to 22,000 (FWHM) in spectra shown in Figure 5. Mass calibration may be different by as much as 250 ppm between the two modes, however, after recalibration, the average mass measurement error for both

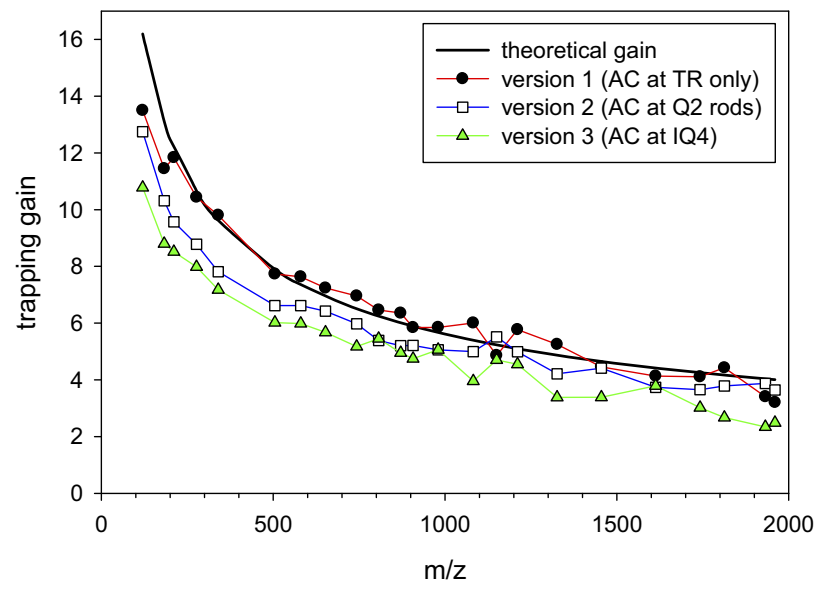

Figure 6. The plot of Zeno pulsing gain versus $m / z$ of ion: theory and experiment, versions 1, 2, and 3. Each curve represents the average over three experiments performed under slightly different conditions. compounds tested remains almost the same and stays within 1 ppm (RMS), when not limited by ion statistics or peak interference (see Table 1). There is a weak trend indicating that mass accuracy improves when both $\mathrm{rf}$ and $\mathrm{AC}$ frequencies are increased, like it happened in transition from version 1 to 2 . One possible explanation for this effect is that ions of differing $m / z$ leave the trap at different phases of $\mathrm{AC}$, which has a small but measurable effect on their starting conditions in the TOF extraction region. The difference in starting conditions then translates into a mass measurement error. This effect averages out better at higher $\mathrm{AC} / \mathrm{rf}$ frequencies. For a similar reason mass measurement error is larger for version 3: ions are affected by AC even after they leave the trap and are accelerated towards the TOF entrance. Since heavy ions $(m / z>800)$ escape the trap at higher AC amplitudes, their mass accuracy is degraded more in this version, as is evident from Table 1 . At the same time, mass resolution was reduced by $10 \%$ to $15 \%$ for some of the peaks at high $m / z$.

The only instrumental parameter that suffers significantly as a result of using the described method is dynamic range. Simultaneously with 3 - to 14 -fold increase of intensities the repetition rate of TOF extractions is dropped by a factor of $\sim 15$, which means that intensities per peak per TOF transient can go up by 200 times, often resulting in saturation of large peaks in the detection system. In our instrument, even with a multichannel TDC, the use of Zeno pulsing is limited to total ion currents (TIC) below $10^{5}$ counts/s if qualitative information is required. Several strategies such as turning off Zeno pulsing for intense ion beams and the use of ADC instead of TDC can be employed to enable recording of stronger ion currents and to extend the dynamic range of the instrument. At $\mathrm{TIC}>10^{6}$ counts/s, at some point the adverse effect of the space charge overfilling the trap may start damaging performance of the setup. However, in the present system, this effect is completely masked by the TDC saturation, which occurs earlier.

\section{Conclusions}

A new method for duty cycle improvement of orthoTOF mass spectrometers has been developed and tested. The method essentially eliminates the largest channel of ion losses characteristic for this type of analyzers. It enables almost $100 \%$ duty cycle over a wide $\mathrm{m} / \mathrm{z}$-range from 120 to 2000 , resulting in sensitivity gains from 3 to 14 without loss of mass accuracy or resolution. Due to reduced linear dynamic range, the application strategy may involve using this method in MS/MS only, where intensities are in general several orders of magnitude lower than in TOF MS, and where an average gain of 7 is more valuable. The switching time between the normal and Zeno pulsing mode is in the millisecond range, which enables its use "on demand" in information-dependent acquisitions, for example in those MS/MS experiments that are triggered 
Table 1. Mass error (RMS) in different operational modes

\begin{tabular}{lcc}
\hline \multicolumn{1}{c}{ Modelmass error } & $\begin{array}{c}\Delta m / m, \mathrm{ppm} \\
\text { all peaks }\end{array}$ & $\begin{array}{c}\Delta m / m, \mathrm{ppm} \\
\mathrm{m} / \mathrm{z}>800\end{array}$ \\
\hline \hline Continuous injection & 0.6 & 0.4 \\
Version 1 (AC at trap only) & 0.8 & 1.1 \\
Version 2 (AC at main rods) & 0.35 & 0.2 \\
Version 3 (AC at IO4) & 1.0 & 1.55 \\
\hline
\end{tabular}

by low intensity precursors in single MS experiments. In such cases, another set of TOF calibration coefficients should be applied.

In the second implementation with no small segments in Q2 and AC voltage applied to the main rods (version 2), the measured sensitivity gains were typically $88 \%$ of those predicted by the theory. An even simpler set-up of version 3 with AC voltage applied to the exit gate IQ4 creates a similar potential distribution inside the trap. However, in this configuration, AC voltage affects ions after they exit the trap, thus somewhat reducing the duty cycle gain $(76 \%$ of theoretical), mass resolution and accuracy.

\section{Acknowledgments}

The authors thank Slobodan Marinkovic and Pablo Dominguez for mechanical design, Nic Bloomfield and Min Yang for software support, and Bruce Thomson and Bob Haufler for valuable discussions.

\section{References}

1. Dodonov, A. F.; Chernushevich, I. V.; Laiko, V. V. Electrospray Ionization on a Reflecting Time-of-Flight Mass Spectrometer. In Time-of-Flight
Mass Spectrometry, Cotter, R. J.; Ed.; Am. Chem. Soc. Symposium Series 549: Washington, DC, 1994; p. 108-123.

2. Verentchikov, A. N.; Ens, W.; Standing, K. G. Reflecting Time-of-Flight Mass Spectrometer with an Electrospray Ion Source and Orthogonal Extraction. Anal. Chem. 1994, 66, 126-133.

3. Guilhaus, M.; Selby, D.; Mlynski, V. Orthogonal Acceleration Time-ofFlight Mass Spectrometry. Mass Spectrom. Rev. 2000, 19, 65-107.

4. Chernushevich, I. V.; Ens, W.; Standing, K. G. Orthogonal Injection TOF MS for Analyzing Biomolecules. Anal. Chem. 1999, 71, 452A-461A.

5. Morris, H. R.; T. Paxton, T.; Dell, A.; Langhorne, J.; Berg, M.; Bordoli, R. S.; Hoyes, J.; Bateman, R. H. High Sensitivity Collisionally-Activated Decomposition Tandem Mass Spectrometry on a Novel Quadrupole/ Orthogonal-Acceleration Time-of-Flight Mass Spectrometer. Rapid Commun. Mass Spectrom. 1996, 10, 889-896.

6. Chernushevich, I. V.; Loboda, A. V.; Thomson, B. A. An Introduction to Quadrupole-Time-of-Flight Mass Spectrometry. J. Mass Spectrom. 2001, 36, 849-865.

7. Whitehouse, C. M.; Guilcicek, E.; Andrien, B.; Banks, F.; Mancini, R. A Two-Dimensional Ion Trap API TOF Mass Spectrometer. Proceedings of the 46th ASMS Conference on Mass Spectrometry and Allied Topics; Orlando, FL, May 3-June 4, 1998; p. 39.

8. Chernushevich, I. V. Duty Cycle Improvement for a QqTOF Mass Spectrometer and Its Use for Precursor Ion Scanning. Eur. Mass Spectrom. 2000, 6, 471-479.

9. Brenton, A. G.; Krastev, T.; Rousell, D. J.; Kennedy, M. A.; Craze, A. S. Williams, C. M. Improvement of the Duty Cycle of an Orthogonal Acceleration Time-of-Flight Mass Spectrometer Using Ion Gates. Rapid Commun. Mass Spectrom. 2007, 21, 3093-3102.

10. Okumura, A.; Hirabayashi, A.; Baba, T.; Hashimoto, Y.; Waki, I.; Yoshinari, K. Orthogonal Trap-TOF Mass Spectrometer (1)-Synchronous Coupling of Trap and TOF. Proceedings of the 51st ASMS Conference on Mass Spectrometry and Allied Topics; Montreal, Quebec, Canada, June 8-12, 2003.

11. Hashimoto, Y.; Hasegawa, H.; Satake, H.; Baba, T.; Waki, I. Duty Cycle Enhancement of an Orthogonal Acceleration Mass Spectrometer Using an Axially-Resonant Excitation Linear Ion Trap. J. Am. Soc. Mass Spectrom. 2006, 17, 1669-1674.

12. Loboda, A. V.; Krutchinsky, A.; Loboda, O.; McNabb, J.; Spicer, V.; Ens, W.; Standing, K. G. Novel Linac II Electrode Geometry for Creating an Axial Field in a Multipole Ion Guide. Eur. Mass Spectrom. 2000, 6, 531-536.

13. Gerlich, D. Inhomogeneous RF Fields: A Versatile Tool for the Study of Processes with Slow Ions. In State-selected and State-to-State Ion-Molecule Reaction Dynamics. Part 1, Ng, C.-Y.; Baer, M.; Eds.; John Wiley and Sons: New York, 1992; p. 1-177.

14. http://en.wikipedia.org/wiki/Zeno's_paradoxes\#Achilles_and_the tortoise. 